\title{
Short communication: Modulation of the small intestinal microbial community composition over short-term or long-term administration with Lactobacillus plantarum ZDY2013
}

\author{
Qiong Xie, ${ }^{*}$ Mingfang Pan, $†$ Renhui Huang, ${ }^{*}$ Ximei Tian, $†$ Xueying Tao, ${ }^{*}$ Nagendra P. Shah,‡ Hua Wei, ${ }^{\dagger} \dagger$ \\ and Cuixiang Wan* ${ }^{1}$ \\ *State Key Laboratory of Food Science and Technology, Nanchang University, Nanchang 330047, P. R. China \\ †College of Life Science, Nanchang University, Nanchang 330031, P. R. China \\ ¥Food and Nutritional Science, School of Biological Sciences, the University of Hong Kong, Pokfulam Road, Hong Kong
}

\begin{abstract}
The small intestinal (SI) microbiota has an essential role in the maintenance of human health. However, data about the indigenous bacteria in SI as affected by probiotics are limited. In our study, the short-term and long-term effects of a probiotic candidate, Lactobacillus plantarum ZDY2013, on the SI microbiota of $\mathrm{C} 57 \mathrm{BL} / 6 \mathrm{~J}$ mice were investigated by the Illumina HiSeq (Novogene Bioinformatics Technology Co., Ltd., Tianjin, China) platform targeting the $\mathrm{V} 4$ region of the $16 \mathrm{~S}$ rDNA. A total of 858,011 sequences in 15 samples were read. The $\alpha$ diversity analysis revealed that oral administration with L. plantarum ZDY2013 for $3 \mathrm{wk}$ led to a significant increase in the richness and diversity of the SI bacterial community. Principal coordinate analysis and unweighted pair-group method with arithmetic means analysis showed a clear alteration in the SI microbiota composition after 3 wk of L. plantarum ZDY2013 treatment, although these changes were not found 6 wk after ceasing L. plantarum ZDY2013 administration. Species annotation showed that the dominant phyla in SI microbiota were Firmicutes, Bacteroidetes, Proteobacteria, and Verrucomicrobia. Interestingly, operational taxonomic unit cluster analysis showed that administration with L. plantarum ZDY2013 for $3 \mathrm{wk}$ significantly increased the abundance of Proteobacteria, but decreased that of Bacteroidetes. Linear discriminant analysis coupled with effect size identified 18 bacterial taxa (e.g., Ruminococcus spp. and Clostridium spp.) that overgrew in the SI microbiota of the mice administered with L. plantarum ZDY2013 for $3 \mathrm{wk}$, and most of them belonged to the phyla Bacteroidetes and Proteobacteria. However, only one bacterial taxon (e.g., Nocardioides spp.) was over-represented in the SI
\end{abstract}

Received March 6, 2016

Accepted May 19, 2016.

${ }^{1}$ Corresponding author: cuixiangwan@ncu.edu.cn microbiota of mice 6 wk after L. plantarum ZDY2013 administration. Overall, this study shows that oral administration with probiotic results in an important but transient alteration in the microbiota of SI.

Key words: small intestinal microbiota, Lactobacillus plantarum ZDY2013, Illumina HiSeq, linear discriminant analysis coupled with effect size

\section{Short Communication}

The human gastrointestinal (GI) tract contains several compartments with distinct anatomy and function (Bernet et al., 1994). The main role of the GI tract is to supply the body with essential nutrients. The GI tract harbors at least 100 trillion $\left(10^{14}\right)$ microbial cells (Whitman et al., 1998) and plays a profound role in host physiology and health (Virgin and Todd, 2011; Clemente et al., 2012). The distal GI tract microbiota, often represented as fecal microbiota, harbor $10^{10}$ to $10^{11}$ bacterial cells $/ \mathrm{g}$. Accumulative data suggested that a potential link exists between microbiota and status of health or disease (Kurokawa et al., 2007; Turnbaugh et al., 2009; Qin et al., 2010). A major drawback of the use of fecal samples to determine the intestinal microbial composition is that fecal microbiota represents only the end of the colon, leaving other parts of the GI tract, particularly the small intestine (SI), unexplored. The SI is a harsh environment for microbial life containing relative low density of flora $\left(10^{2}-10^{7}\right.$ cells $\left./ \mathrm{g}\right)$ because of the short transit time, and excretion of digestive enzymes and bile, thereby requiring different survival strategies for microbes compared with those residing in the colon (Bernet et al., 1994; Leser and Mølbak, 2009). However, the SI microbiota might also have a profound effect on various aspects of the host's physiology, including immune, metabolic, and endocrine functions (Cotter, 2011; Raoult and Henrissat, 2014). Data showed that the composition of the microbiota is relatively stable within healthy adult individuals (Costello 
et al., 2009; Caporaso et al., 2011). However, studies showed that imbalances of the microbiota composition in SI were associated with pediatric celiac disease (Hathout et al., 2011), inflammatory bowel disease (Alakomi et al., 2000), irritable bowel syndrome (Makras et al., 2006; Reyes et al., 2010), and pouchitis (Aiba et al., 1998; Zou et al., 2009; Dominici et al., 2011). Dietary intervention in particular had been shown to have significant effects on the changes in microbiota (Reyes et al., 2010). Hence understanding the influence of the endogenous microbiota by intervention is an important step for developing therapies to correct gut dysbiosis.

Probiotics are defined as "live microorganisms, which when administered in adequate amounts, may have a positive effect to alter the microflora of host and exert a health benefit to the host" (Schwiertz et al., 2010). Several studies disclosed that intervention by probiotics could combat several GI diseases, such as irritable bowel syndrome (Ushiyama et al., 2003) and infectious diarrhea (Basu et al., 2011). Moreover, it was reported that probiotics from human resources might perform better in the gut environment (Marco et al., 2010) and may persist for several days or weeks (Saxelin et al., 2010; Smith et al., 2011). On the other hand, a few publications mentioned that ingestion of probiotics does not result in global and long-lasting changes to the intestinal microbiome of healthy adult individuals (McNulty et al., 2011; Kim et al., 2013; Lahti et al., 2013). The above paradox pertaining to colonization of probiotics attracted much attention for the evaluation of probiotic strains, especially for novel strains isolated from fermented foods. To our knowledge, short-term or long-term effect of probiotics on gut depends on many factors such as species of probiotics, dosage of administration, or consumption period.

Lactobacillus plantarum ZDY2013, a novel strain isolated from fermented beans, was proven to have a wide range of beneficial properties, including antioxidant abilities, resistance to high acid stress and antimicrobial activities in vitro (Huang et al., 2015), and immune regulatory abilities and gastric microbiota modulation properties in vivo (Pan et al., 2015). Considering that the selective modulation of the intestinal microbiota through orally administration of probiotics is a developing strategy to maintain human health or even combat diseases, the objective of this study was to evaluate the effect of L. plantarum ZDY2013 administration on SI microbiota composition over short-term or long-term intervention. The protocol for carrying out animal experiments was approved by Nanchang University Animal Ethics Committee. All the animal experiments were approved by the Animal Care Review Committee (approval number 0064257) of Nanchang University (Jiangxi, China). Pathogen-free C57BL/6J female mice (SJA Laboratory Animal Co., Ltd., Hunan, China) were kept at $24 \pm 2^{\circ} \mathrm{C}$ and $40 \pm 20 \%$ relative humidity with a 12-h lighting cycle for $1 \mathrm{wk}$ with free access to food and water. After 1 wk of acclimatization, all twenty-four 6-wk-old female mice were randomly assigned into 2 groups, the experimental group ( $\mathrm{n}=$ 16) and control group $(n=8)$. For the experimental group, sixteen 6-wk-old mice were intragastrically administered with $400 \mu \mathrm{L}$ of $L$. plantarum ZDY2013 at a concentration of $2.0 \times 10^{9} \mathrm{cfu} / \mathrm{mL}$ per mouse daily for $3 \mathrm{wk}$. For the control group, mice were intragastrically administered with $400 \mu \mathrm{L}$ of saline $(0.8 \% \mathrm{wt} / \mathrm{vol})$ per mouse daily. Simultaneously, activity, behavior, fur health, and live weight were observed twice daily during the experimental period. Half of the mice from the experimental group were killed by cervical dislocation after 3 wk of L. plantarum ZDY2013 administration (n $=8$, LP3), and the rest were killed 6 wk after stopping L. plantarum ZDY2013 administration $(\mathrm{n}=8$, LP9). Two centimeters of intestine above the cecum of each mouse was collected aseptically and immediately transferred into a liquid nitrogen container, and then stored at $-80^{\circ} \mathrm{C}$ for SI microbiota analysis. The fecal samples were collected simultaneously and the viable L. plantarum ZDY2013 population was analyzed by real-time quantitative PCR (Huang et al., 2015). The results showed that the relative abundance of $L$. plantarum ZDY 2013 was 37.35-fold in LP3 when compared with the initial value, but it was no longer detected after stopping L. plantarum ZDY2013 feeding for $6 \mathrm{wk}$ (Supplemental Figure S1; http://dx.doi.org/10.3168/ jds.2016-11141).

Total bacteria DNA was extracted from the SI samples using the SDS method (Yu and Morrison, 2004). The DNA concentration and purity were examined by agarose gel electrophoresis and spectrophotometry using the NanoDrop (Thermo Scientific, Wilmington, $\mathrm{DE})$. According to the concentration, DNA was diluted to $1 \mathrm{ng} / \mu \mathrm{L}$ using sterile water and stored at $-80^{\circ} \mathrm{C}$ for further analysis. The sequences of the primers targeting the V4 hyper-variable region of the bacterial 16S rRNA

Table 1. Number of total tags, operational taxonomic units (OTU), and estimated sample coverage for 16S rRNA libraries of LP3, LP9, and control groups $(\text { mean } \pm \mathrm{SD})^{1}$

\begin{tabular}{lccc}
\hline Samples & Total tags & OTU $^{2}$ & Coverage (\%) \\
\hline Control & $58,799 \pm 4,685$ & $957 \pm 182$ & $99.62 \pm 0.08$ \\
LP3 & $55,485 \pm 7,071$ & $1,395 \pm 263^{*}$ & $99.62 \pm 0.15$ \\
LP9 & $57,318 \pm 5,673$ & $935 \pm 133$ & $99.62 \pm 0.05$ \\
\hline
\end{tabular}

${ }^{1}$ LP3 $=$ mice were killed after 3 wk of Lactobacillus plantarum ZDY2013 administration; LP9 $=$ mice were killed 6 wk after stopping $L$. plantarum ZDY2013 administration.

${ }^{2}$ The OTU were defined at the $97 \%$ similarity level.

$* P<0.05$. 
genes were as follows: 515f (5'-GTGCCAGCMGCCGCGGTAA-3') and 806r (5'-GGACTACHVGGGTWTCTAAT- $3^{\prime}$ ), where $\mathrm{f}$ is forward and $\mathrm{r}$ is reverse. To generate an amplicon size of $253 \mathrm{bp}$, reverse primers contained a 6-bp error-correcting barcode which is unique to each sample. A PCR reaction was performed using Phusion High-Fidelity PCR Master Mix (New England Biolabs, Ipswich, MA) with GC Buffer with the following conditions: $94^{\circ} \mathrm{C}$ for $3 \mathrm{~min}$ ( 1 cycle); fol- lowed by 35 cycles at $94^{\circ} \mathrm{C}$ for $45 \mathrm{~s}, 50^{\circ} \mathrm{C}$ for $60 \mathrm{~s}, 72^{\circ} \mathrm{C}$ for $90 \mathrm{~s}$; and a last step of $72^{\circ} \mathrm{C}$ for $10 \mathrm{~min}$. The PCR products were purified by using the Qiagen gel extraction kit (Qiagen, Hilden, Germany) and sequenced by the Illumina HiSeq 2500 PE250 platform (Novogene Bioinformatics Technology Co., Ltd., Tianjin, China).

Bioinformatic analysis of sequencing data was conducted using quantitative insights into microbial ecology (QIIME; Caporaso et al., 2010). The low-quality
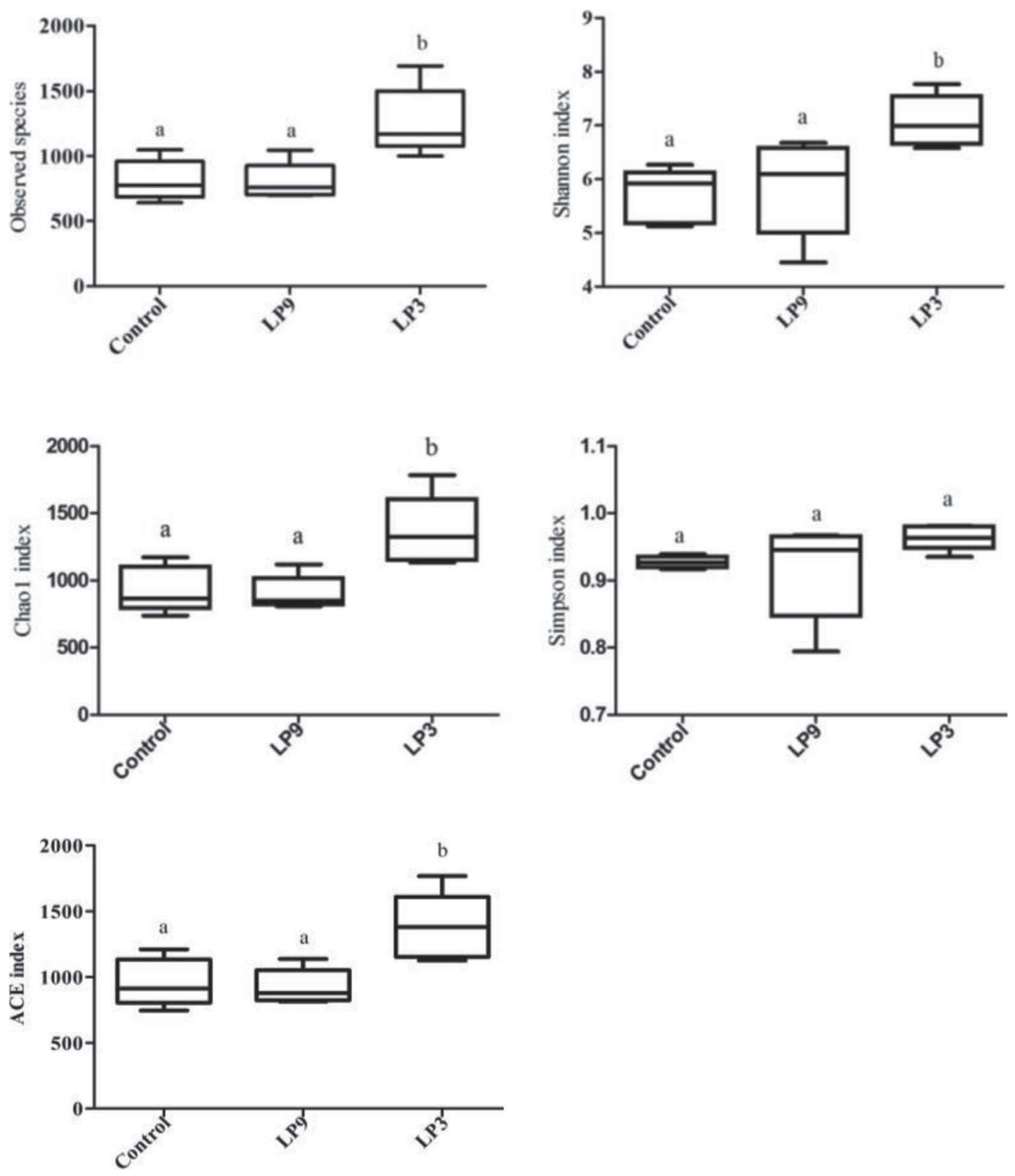

Figure 1. Differences in bacterial community richness and diversity between LP3, LP9, and control groups. LP3 = mice killed after 3 wk of Lactobacillus plantarum ZDY2013 administration; LP9 = mice killed 6 wk after stopping L. plantarum ZDY2013 administration. ACE: the index used to estimate the community operational taxonomic unit number. Significant differences between bars are denoted by a different letter (a,b; $P<0.001)$. Each value is expressed as mean $\pm \mathrm{SD}$. 
reads, barcodes and primers, and chimera sequences were removed using the UCHIME software based on the UCHIME algorithm and the effective tags finally obtained. Sequences were clustered into operational taxonomic units (OTU) with a $97 \%$ similarity threshold. Sequence was picked as a representative for each OTU, and the RDP Classifier (version 2.2, http:// sourceforge.net/projects/rdp-classifier/) was used to annotate taxonomic information for each representative sequence. The OTU were used for $\alpha$ diversity analysis including observed species, Chao1, ACE, and Shannon and Simpson indexes, which were generated with
QIIME (Version 1.7.0) and displayed with $\mathrm{R}$ software (Version 2.15.3). Jackknifed $\beta$ diversity contained unweighted Unifrac distances were calculated by QIIME software (Version 1.7.0). Consequently, these distances were visualized by principal coordinate analysis and unweighted pair-group method with arithmetic means (UPGMA). Principal coordinate analysis helps to get principal coordinates and visualize them from complex multidimensional data. The UPGMA clustering is a type of hierarchical clustering method using average linkage and can be used to interpret the distance matrix.

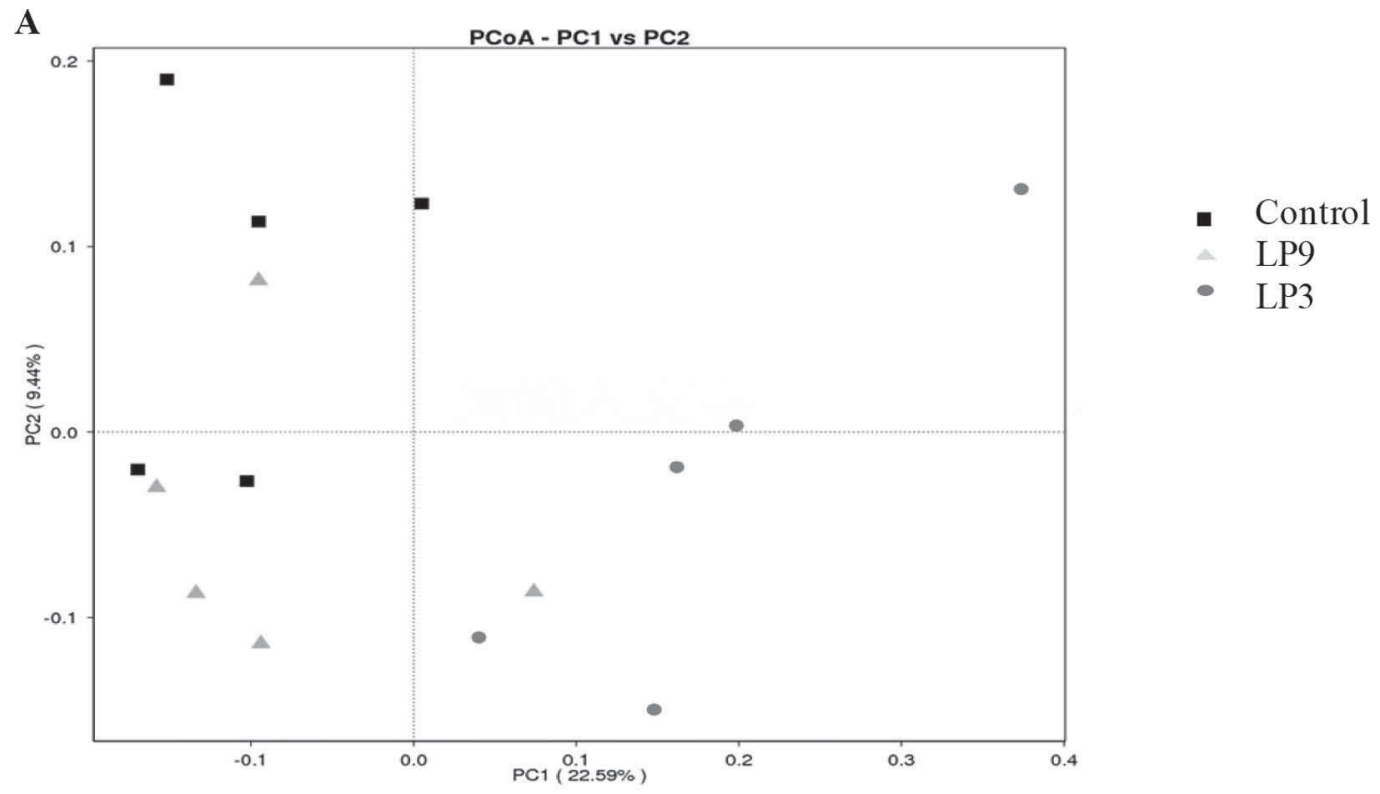

\section{B}

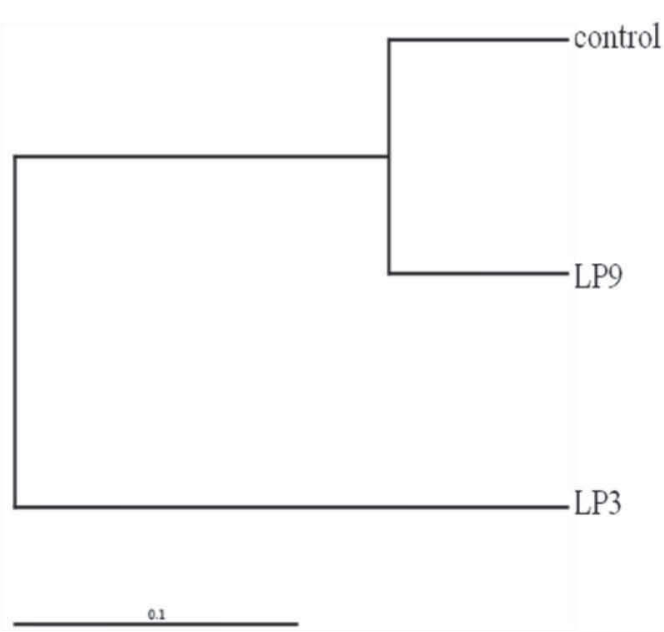

Figure 2. (A) Principal coordinate analysis (PCoA) of the dissimilarity between the small intestinal (SI) microbiota: UNWeighted UniFrac $\mathrm{PCoA}$ plotted against the PC1 versus $\mathrm{PC} 2$ axes. The percentages indicate the relative contribution of the 2 principal components (PC1-PC2). (B) Unweighted pair-group method with arithmetic means (UPGMA) clustering of the SI microbiota. The clustering tree structure based on unweighted UniFrac. 
Data were expressed by mean values \pm SEM. Oneway ANOVA test was used for significance testing by GraphPad Prism version 5.01 (GraphPad Software Inc., La Jolla, CA). Linear discriminant analysis coupled with effect size (LEfSe) was performed to identify the bacterial taxa differentially represented between groups at genus or higher taxonomy levels.

A total of 858,011 effective sequences were used for abundance and diversity analyses as well as taxonomic comparisons. Principally, as shown in Table 1, sequences were delineated into different OTU by a similarity value of $97 \%$, and thus a total of 16,429 OTU were obtained with a range of 772 to 1,475 OTU in each group. The numbers of OTU of LP3 group were significantly higher than that of control, but no difference was found between LP9 and LP3 or the control. The average coverage was as high as $99.62 \%$, indicating that the majority of bacterial phenotypes present in the samples were identified in this study. The $\alpha$ diversity index, including observed-species, Chao1, ACE, and Shannon and Simpson indexes, gives a basic data of the bacterial richness and diversity within each sample. As shown in Figure 1, all indexes except for Simpson significantly $(P<0.01)$ increased in the LP3 group when compared with the control group, whereas these significant changes were not observed in LP9 $(P>0.05)$. All the results indicated that $3 \mathrm{wk}$ of $L$. plantarum ZDY2013 administration improved the SI microbiota richness and diversity, though these improvements appeared to be instant. Recent studies showed that an ecosystem with high richness and diversity species can maintain system stability and balance due to its stronger antidisturbance ability against external environmental interference (Allesina and Tang, 2012).

To further confirm the effect of L. plantarum ZDY2013 on the overall composition of SI microbial communities, principal coordinate analysis based on unweighted Unifrac distance matrixes was also employed. As shown in Figure 2A, a clear shift in the SI microbial composition between LP3 and control was observed, with principal component 1 (PC1) accounting for $22.59 \%$ of the total variation, whereas the majority of the bacterial communities among LP9 and control were quite similar. The UPGMA analysis (Figure 2B) confirmed the above results. It can be concluded that 3 wk of L. plantarum ZDY2013 administration altered the overall bacterial composition in SI. However, this change was not long term, as 6 wk postcessation of administration, the SI microbiota tended to return to normal.

Taxonomy comparisons at phylum level showed that the dominant phyla in SI microbiota were Bacteriodetes, Firmicutes, Proteobacteria, and Verrucomicrobia (Figure 3). The LP3 contained a larger population of Proteobacteria $(26.40 \pm 15.94 \%)$ and less Bacteroide-

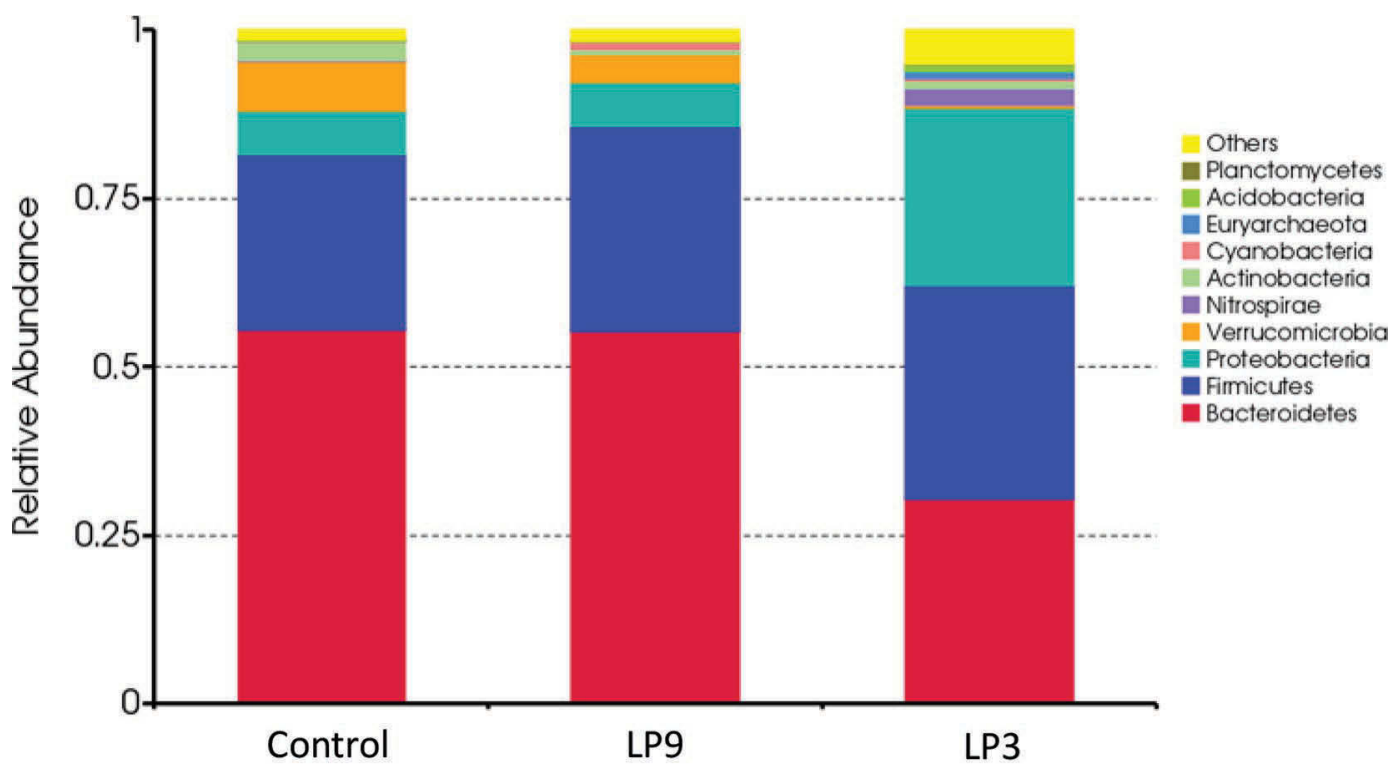

Group Name

Figure 3. Bacterial composition of the different communities in the small intestine (SI) of mice (percent of the relative read abundance of top 10 bacterial phyla within each community). Each bar represents the average relative abundance of bacterial taxa within a group. LP3 $=$ mice killed after 3 wk of Lactobacillus plantarum ZDY2013 administration; LP9 = mice killed 6 wk after stopping L. plantarum ZDY2013 administration. Color version available online. 
A

\section{Control $\square$ LP.3}
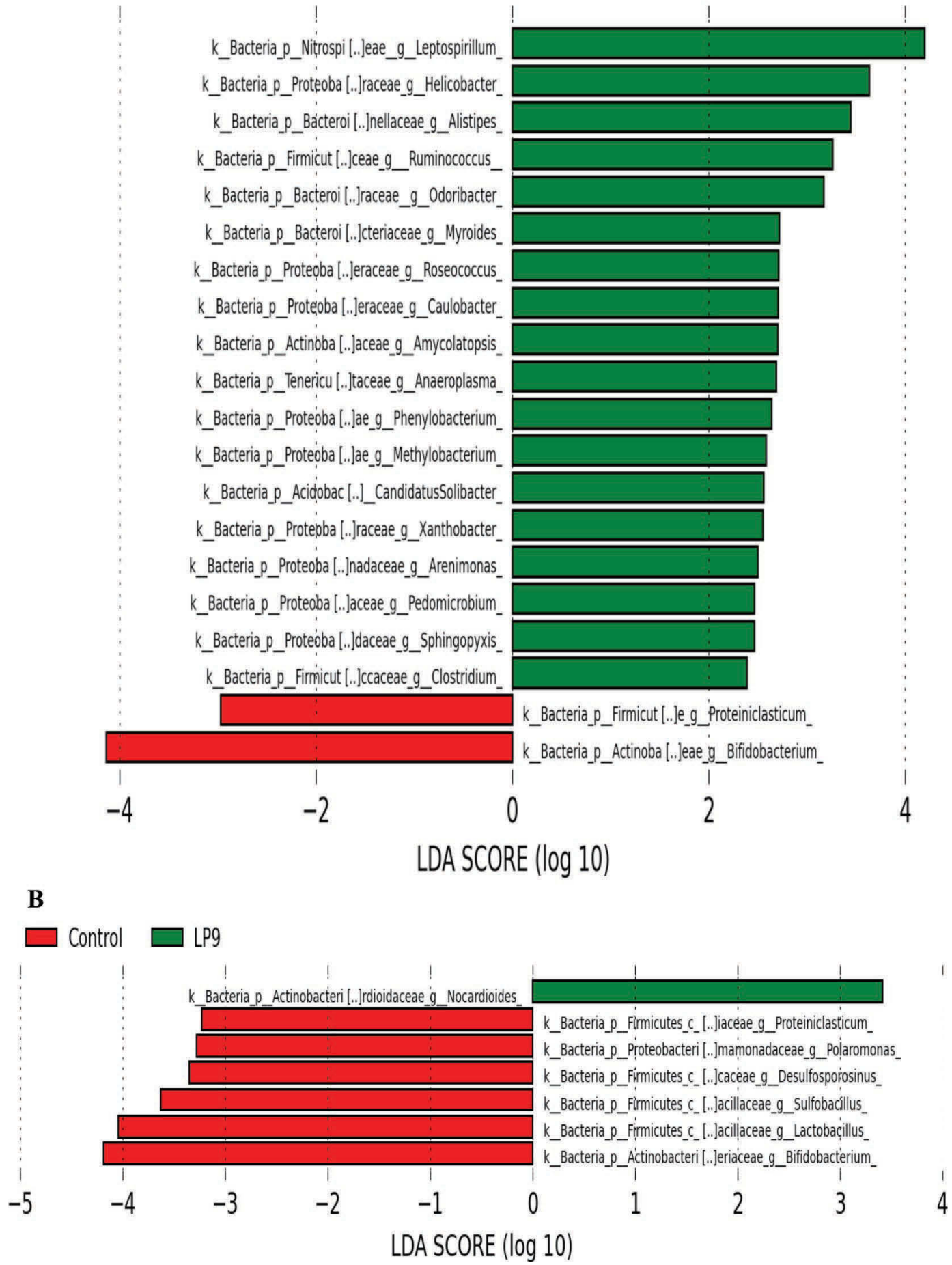

Figure 4. Bacterial taxa significantly differentiated between the control and LP3 (A) or LP9 (B) groups were identified by linear discriminant analysis (LDA) coupled with effect size using the default parameters. LP3 = mice killed after 3 wk of Lactobacillus plantarum ZDY2013 administration; LP9 = mice killed 6 wk after stopping L. plantarum ZDY2013 administration. Color version available online. 
tes $(30.33 \pm 20.02 \%)$ than that of the control $(6.51$ $\pm 4.82 \%$ and $55.51 \pm 3.17 \%$, respectively), and this difference was no longer found in LP9.

To further analyze the special bacteria taxa associated with L. plantarum ZDY2013 administration, LEfSe was used to compare the bacterial taxa abundance between the LP3 and control group. As shown in Figure 4A, 18 bacterial taxa overgrew in mice after 3 wk of L. plantarum ZDY2013 administration; among them Helicobacter spp., Alistipes spp., Ruminococcus spp., Myroides spp., and Clostridium spp. showed an obvious change. Ruminococcus spp., which belongs to the phylum Firmicutes, can generate short-chain fatty acids (Pryde et al., 2002), which may enhance the protection of the intestinal barrier and reduce the colonization of opportunistic pathogens in the intestines (Chassard and Bernalier Donadille, 2006). This genera was also found in long-living elderly healthy people in our previous study (Yu et al., 2015), and increased in the feces of mice following the consumption of the phytonutrient eugenol (Wlodarska et al., 2015). Alistipes spp. and Myroides spp. belonged to the phylum Bacteriodetes; the former produces succinic acid as the principal metabolic end product of glucose fermentation, and iso$\mathrm{C}_{15: 0}$ as its major long-chain cellular fatty acid (Rautio et al., 2003), whereas the latter was able to emulsify crude oil and use aliphatic hydrocarbons or aromatic hydrocarbons as sole carbon sources (Mukai et al., 2002). Hence, we hypothesized that these 2 genera were able to promote digestion and absorption of food. Clostridium spp., which belonged to the phylum Firmicutes, can use a wide range of carbon sources (e.g., glucose, galactose, cellobiose, mannose, xylose, and arabinose) for increasing the production of short-chain fatty acids (Ezeji et al., 2007; Lee et al., 2008), subsequently thickening the inner mucus layer (Deplancke et al., 2002; Kaewnopparat et al., 2013), and thus preventing the invasion of pathogens in the intestines (Kurokawa et al., 2007; Johansson et al., 2013). Interestingly, some genera of opportunistic pathogens (e.g., Helicobacter spp.), belonging to Proteobacteria, were found in the LP3 group. Some species belong to Helicobacter spp. (e.g., Helicobacter pylori), which is known to induce gastritis although a recent report showed that it has an effect on conferring protection against tuberculosis (Perry et al., 2010). On the contrary, some Helicobacter spp., such as Helicobacter bilis have proven to attenuate gastric infection (Lemke et al., 2009). However, the relative abundance of this genus was less than $0.01 \%$ in all 3 groups, although we could not identify it at the species level.

Compared with the short-term effect posed by L. plantarum ZDY2013, LP9 presented a recovery tendency in the comparative analysis of LEfSe with the control. As shown in Figure 4B, only one taxon, Nocardioides spp., overgrew in the LP9 group (Nocardioides spp.). Nocardioides spp. belonged to the phylum Actinobacteria and was reported to be isolated from soil or healthy rabbit feces, and its secondary metabolites (such as antibiotic N-(2-hydroxyphenyl)-2-phenazinamine) had antimicrobial and antifungal activities (Lee et al., 2012; Zhang et al., 2015).

Recently, with the accumulation of microbiota data by the intervention of probiotics (e.g., Lactobacillus, Bifidobacterium, Enterococcus, and Pediococcus; El Aidy et al., 2015; Wu et al., 2016), selective modulation of the intestinal microbiota through oral administration of probiotics (e.g., L. plantarum and Bifidobacterium) has become a developing strategy to maintain human health or even treat diseases (Fuentes et al., 2008). In our previous parallel assay against $H$. pylori infection, we discovered that pretreatment with a novel strain of L. plantarum ZDY2013 played an important role in altering gastric microbiota and preventing gastric mucosal inflammation. In this study, we investigated the short-term and long-term effects of L. plantarum ZDY2013 on the composition of SI microbiota using healthy mice as a model. We demonstrated that consumption of L. plantarum ZDY2013 for 3 wk resulted in observable changes in the SI microbiota, including increased bacterial richness and diversity, and distinct alteration in bacterial community composition. However, this modulation induced by L. plantarum ZDY2013 was transient and not long term, suggesting that oral administration of L. plantarum ZDY2013 should be long term to confer a beneficial effect on the host's health and potential use for the treatment/prevention of Helicobacter pylori-induced gastric inflammation.

\section{ACKNOWLEDGMENTS}

This study was sponsored by the National Natural Science Foundation of China (grant no. NSF 31360377), Academic and Technical Leaders Training Program for Major Subjects of Jiangxi Province (2009; China), Ganpo Talent 555 Engineering Project of Jiangxi Province (China), and Jiangxi Provincial Education Department (GJJ3098; China).

\section{REFERENCES}

Alakomi, H.-L., E. Skyttä, M. Saarela, T. Mattila-Sandholm, K. Latva-Kala, and I. Helander. 2000. Lactic acid permeabilizes gramnegative bacteria by disrupting the outer membrane. Appl. Environ. Microbiol. 66:2001-2005.

Allesina, S., and S. Tang. 2012. Stability criteria for complex ecosystems. Nature 483:205-208.

Aiba, Y., N. Suzuki, A. Kabir, A. Takagi, and Y. Koga. 1998. Lactic acid-mediated suppression of Helicobacter pylori by the oral ad- 
ministration of Lactobacillus salivarius as a probiotic in a gnotobiotic murine model. Am. J. Gastroenterol. 93:2097-2101.

Basu, P. P., K. Rayapudi, T. Pacana, N. J. Shah, N. Krishnaswamy, and M. Flynn. 2011. A randomized study comparing levofloxacin, omeprazole, nitazoxanide, and doxycycline versus triple therapy for the eradication of Helicobacter pylori. Am. J. Gastroenterol. 106:1970-1975.

Bernet, M.-F., D. Brassart, J. Neeser, and A. Servin. 1994. Lactobacillus acidophilus LA 1 binds to cultured human intestinal cell lines and inhibits cell attachment and cell invasion by enterovirulent bacteria. Gut 35:483-489.

Caporaso, J. G., J. Kuczynski, J. Stombaugh, K. Bittinger, F. D. Bushman, E. K. Costello, N. Fierer, A. G. Pena, J. K. Goodrich, and J. I. Gordon. 2010. QIIME allows analysis of high-throughput community sequencing data. Nat. Methods 7:335-336.

Caporaso, J. G., C. L. Lauber, E. K. Costello, D. Berg-Lyons, A. Gonzalez, J. Stombaugh, D. Knights, P. Gajer, J. Ravel, and N. Fierer. 2011. Moving pictures of the human microbiome. Genome Biol. 12:R50.

Chassard, C., and A. Bernalier Donadille. 2006. $\mathrm{H}_{2}$ and acetate transfers during xylan fermentation between a butyrate-producing xylanolytic species and hydrogenotrophic microorganisms from the human gut. FEMS Microbiol. Lett. 254:116-122.

Clemente, J. C., L. K. Ursell, L. W. Parfrey, and R. Knight. 2012 The impact of the gut microbiota on human health: An integrative view. Cell 148:1258-1270.

Costello, E. K., C. L. Lauber, M. Hamady, N. Fierer, J. I. Gordon, and R. Knight. 2009. Bacterial community variation in human body habitats across space and time. Science 326:1694-1697.

Cotter, P. D. 2011. Small intestine and microbiota. Curr. Opin. Gastroenterol. 27:99-105.

Deplancke, B., O. Vidal, D. Ganessunker, S. M. Donovan, R. I. Mackie, and H. R. Gaskins. 2002. Selective growth of mucolytic bacteria including Clostridium perfringens in a neonatal piglet model of total parenteral nutrition. Am. J. Clin. Nutr. 76:1117-1125.

Dominici, L., M. Moretti, M. Villarini, S. Vannini, G. Cenci, C. Zampino, and G. Traina. 2011. In vivo antigenotoxic properties of a commercial probiotic supplement containing bifidobacteria. Int. J. Probiotics Prebiotics 6:179-186.

El Aidy, S., B. van den Bogert, and M. Kleerebezem. 2015. The smal intestine microbiota, nutritional modulation and relevance for health. Curr. Opin. Biotechnol. 32:14-20.

Ezeji, T. C., N. Qureshi, and H. P. Blaschek. 2007. Bioproduction of butanol from biomass: From genes to bioreactors. Curr. Opin. Biotechnol. 18:220-227.

Fuentes, S., M. Egert, M. Jiménez-Valera, A. Ramos-Cormenzana, A. Ruiz-Bravo, H. Smidt, and M. Monteoliva-Sanchez. 2008. Administration of Lactobacillus casei and Lactobacillus plantarum affects the diversity of murine intestinal lactobacilli, but not the overall bacterial community structure. Res. Microbiol. 159:237-243.

Hathout, A. S., S. R. Mohamed, A. A. El-Nekeety, N. S. Hassan, S. E. Aly, and M. A. Abdel-Wahhab. 2011. Ability of Lactobacillus case and Lactobacillus reuteri to protect against oxidative stress in rats fed aflatoxins-contaminated diet. Toxicon 58:179-186.

Huang, R., X. Tao, C. Wan, S. Li, H. Xu, F. Xu, N. P. Shah, and H. Wei. 2015. In vitro probiotic characteristics of Lactobacillus plantarum ZDY 2013 and its modulatory effect on gut microbiota of mice. J. Dairy Sci. 98:5850-5861.

Johansson, M. E., J. K. Gustafsson, J. Holmén-Larsson, K. S. Jabbar, L. Xia, H. Xu, F. K. Ghishan, F. A. Carvalho, A. T. Gewirtz, H Sjövall, and G. C. Hansson. 2013. Bacteria penetrate the normally impenetrable inner colon mucus layer in both murine colitis models and patients with ulcerative colitis. Gut 63:281-291.

Kaewnopparat, S., N. Dangmanee, N. Kaewnopparat, T. Srichana, M. Chulasiri, and S. Settharaksa. 2013. In vitro probiotic properties of Lactobacillus fermentum SK5 isolated from vagina of a healthy woman. Anaerobe 22:6-13

Kim, S.-W., W. Suda, S. Kim, K. Oshima, S. Fukuda, H. Ohno, H. Morita, and M. Hattori. 2013. Robustness of gut microbiota of healthy adults in response to probiotic intervention revealed by high-throughput pyrosequencing. DNA Res. 20:241-253.
Kurokawa, K., T. Itoh, T. Kuwahara, K. Oshima, H. Toh, A. Toyoda, H. Takami, H. Morita, V. K. Sharma, and T. P. Srivastava. 2007. Comparative metagenomics revealed commonly enriched gene sets in human gut microbiomes. DNA Res. 14:169-181.

Lahti, L., A. Salonen, R. A. Kekkonen, J. Salojärvi, J. Jalanka-Tuovinen, A. Palva, M. Orešič, and W. M. de Vos. 2013. Associations between the human intestinal microbiota, Lactobacillus rhamnosus GG and serum lipids indicated by integrated analysis of highthroughput profiling data. PeerJ 1:e32.

Lee, L.-H., Y.-K. Cheah, S. M. Sidik, N.-S. Ab Mutalib, Y.-L. Tang, H.-P. Lin, and K. Hong. 2012. Molecular characterization of Antarctic actinobacteria and screening for antimicrobial metabolite production. World J. Microbiol. Biotechnol. 28:2125-2137.

Lee, S. Y., J. H. Park, S. H. Jang, L. K. Nielsen, J. Kim, and K. S. Jung. 2008. Fermentative butanol production by Clostridia. Biotechnol. Bioeng. 101:209-228.

Lemke, L. B., Z. Ge, M. T. Whary, Y. Feng, A. B. Rogers, S. Muthupalani, and J. G. Fox. 2009. Concurrent Helicobacter bilis infection in C57BL/6 mice attenuates proinflammatory $H$. pylori-induced gastric pathology. Infect. Immun. 77:2147-2158.

Leser, T. D., and L. Mølbak. 2009. Better living through microbial action: The benefits of the mammalian gastrointestinal microbiota on the host. Environ. Microbiol. 11:2194-2206.

Makras, L., V. Triantafyllou, D. Fayol-Messaoudi, T. Adriany, G. Zoumpopoulou, E. Tsakalidou, A. Servin, and L. De Vuyst. 2006. Kinetic analysis of the antibacterial activity of probiotic lactobacilli towards Salmonella enterica serovar Typhimurium reveals a role for lactic acid and other inhibitory compounds. Res. Microbiol. 157:241-247.

Marco, M. L., M. C. de Vries, M. Wels, D. Molenaar, P. Mangell, S. Ahrne, W. M. de Vos, E. E. Vaughan, and M. Kleerebezem. 2010. Convergence in probiotic Lactobacillus gut-adaptive responses in humans and mice. ISME J. 4:1481-1484.

McNulty, N. P., T. Yatsunenko, A. Hsiao, J. J. Faith, B. D. Muegge A. L. Goodman, B. Henrissat, R. Oozeer, S. Cools-Portier, and G. Gobert. 2011. The impact of a consortium of fermented milk strains on the gut microbiome of gnotobiotic mice and monozygotic twins. Science Translational Medicine 3:106ra106-106ra106.

Mukai, T., T. Asasaka, E. Sato, K. Mori, M. Matsumoto, and H. Ohori. 2002. Inhibition of binding of Helicobacter pylori to the glycolipid receptors by probiotic Lactobacillus reuteri. FEMS Immunol. Med. Microbiol. 32:105-110.

Pan, M., Q. Wu, X. Tao, C. Wan, N. P. Shah, and H. Wei. 2015. Fermentation of Allium chinense bulbs with Lactobacillus plantarum ZDY 2013 shows enhanced biofunctionalities, and nutritional and chemical properties. J. Food Sci. 80:M2272-M2278.

Perry, S., B. C. De Jong, J. V. Solnick, M. de la Luz Sanchez, S. Yang, P. L. Lin, L. M. Hansen, N. Talat, P. C. Hill, and R. Hussain. 2010. Infection with Helicobacter pylori is associated with protection against tuberculosis. PLoS ONE 5:e8804.

Pryde, S. E., S. H. Duncan, G. L. Hold, C. S. Stewart, and H. J. Flint 2002. The microbiology of butyrate formation in the human colon. FEMS Microbiol. Lett. 217:133-139.

Qin, J., R. Li, J. Raes, M. Arumugam, K. S. Burgdorf, C. Manichanh, T. Nielsen, N. Pons, F. Levenez, and T. Yamada. 2010. A human gut microbial gene catalogue established by metagenomic sequencing. Nature 464:59-65.

Raoult, D., and B. Henrissat. 2014. Are stool samples suitable for studying the link between gut microbiota and obesity? Eur. J. Epidemiol. 29:307-309.

Rautio, M., E. Eerola, M.-L. Väisänen-Tunkelrott, D. Molitoris, P. Lawson, M. D. Collins, and H. Jousimies-Somer. 2003. Reclassification of Bacteroides putredinis (Weinberg et al., 1937) in a new genus Alistipes gen. nov., as Alistipes putredinis comb. nov., and description of Alistipes finegoldii sp. nov., from human sources. Syst. Appl. Microbiol. 26:182-188.

Reyes, A., M. Haynes, N. Hanson, F. E. Angly, A. C. Heath, F. Rohwer, and J. I. Gordon. 2010. Viruses in the faecal microbiota of monozygotic twins and their mothers. Nature 466:334-338.

Saxelin, M., A. Lassig, H. Karjalainen, S. Tynkkynen, A. Surakka, H. Vapaatalo, S. Järvenpää, R. Korpela, M. Mutanen, and K. 
Hatakka. 2010. Persistence of probiotic strains in the gastrointestinal tract when administered as capsules, yoghurt, or cheese. Int. J. Food Microbiol. 144:293-300.

Schwiertz, A., D. Taras, K. Schäfer, S. Beijer, N. A. Bos, C. Donus, and P. D. Hardt. 2010. Microbiota and SCFA in lean and overweight healthy subjects. Obesity (Silver Spring) 18:190-195.

Smith, T. J., D. Anderson, L. M. Margolis, A. Sikes, and A. J. Young. 2011. Persistence of Lactobacillus reuteri DSM17938 in the human intestinal tract: Response to consecutive and alternate-day supplementation. J. Am. Coll. Nutr. 30:259-264.

Turnbaugh, P. J., M. Hamady, T. Yatsunenko, B. L. Cantarel, A. Duncan, R. E. Ley, M. L. Sogin, W. J. Jones, B. A. Roe, and J. P. Affourtit. 2009. A core gut microbiome in obese and lean twins. Nature 457:480-484.

Ushiyama, A., K. Tanaka, Y. Aiba, T. Shiba, A. Takagi, T. Mine, and Y. Koga. 2003. Lactobacillus gasseri OLL2716 as a probiotic in clarithromycin-resistant Helicobacter pylori infection. J. Gastroenterol. Hepatol. 18:986-991.

Virgin, H. W., and J. A. Todd. 2011. Metagenomics and personalized medicine. Cell 147:44-56.

Whitman, W. B., D. C. Coleman, and W. J. Wiebe. 1998. Prokaryotes: The unseen majority. Proc. Natl. Acad. Sci. USA 95:6578-6583.
Wlodarska, M., B. P. Willing, D. M. Bravo, and B. B. Finlay. 2015. Phytonutrient diet supplementation promotes beneficial Clostridia species and intestinal mucus secretion resulting in protection against enteric infection. Sci. Rep. 5:9253.

Wu, B.-B., Y. Yang, X. Xu, and W.-P. Wang. 2016. Effects of Bifidobacterium supplementation on intestinal microbiota composition and the immune response in healthy infants. World J. Pediatr. $12: 177-182$.

Yu, X., X. Wu, L. Qiu, D. Wang, M. Gan, X. Chen, H. Wei, and F. Xu. 2015. Analysis of the intestinal microbial community structure of healthy and long-living elderly residents in Gaotian Village of Liuyang City. Appl. Microbiol. Biotechnol. 99:9085-9095.

Yu, Z., and M. Morrison. 2004. Improved extraction of PCR-quality community DNA from digesta and fecal samples. Biotechniques $36: 808-812$.

Zhang, Y., H. Tan, Q. Deng, and L. Cao. 2015. Actinobacterial flora in feces of healthy cottontail rabbits (Sylvilagus auduboni). Probiotics Antimicrob. Proteins 7:9-13.

Zou, J., J. Dong, and X. Yu. 2009. Meta-analysis: Lactobacillus containing quadruple therapy versus standard triple first-line therapy for Helicobacter pylori eradication. Helicobacter 14:97-107. 